\title{
Patienten brauchen bei der Prävention mehr Unterstützung
}

\author{
Karin Kraft \\ Stiftungsprofessur für Naturheilkunde, Universitätsmedizin Rostock, Rostock, Deutschland
}

Im Jahr 2016 wurde die erste repräsentative Umfrage zur Gesundheitskompetenz der deutschen Bevölkerung publiziert [1]. Unter Gesundheitskompetenz ("health literacy") werden das Wissen, die Motivation und die Fähigkeit verstanden, gesundheitsrelevante Informationen ausfindig zu machen, zu verstehen, zu beurteilen und zu nutzen, um die Gesundheit erhalten, sich bei Krankheiten die nötige Unterstützung durch das Gesundheitssystem sichern oder sich kooperativ an der Behandlung und Versorgung beteiligen und die dazu nötigen Entscheidungen treffen zu können [1]. Die Ergebnisse dieser vom Bundesministerium der Justiz und für Verbraucherschutz finanzierten Umfrage bestätigten eine international schon seit über 20 Jahren bekannte Problematik, erregten aber dennoch eine hohe Aufmerksamkeit in gesundheitspolitischen Kreisen. So verfügen nach dieser Umfrage 54,3 Prozent der Deutschen nur über eine eingeschränkte Gesundheitskompetenz. Dieser Teil der Bevölkerung weist nach eigener Einschätzung häufiger einen schlechten Gesundheitszustand auf und hat erhebliche Schwierigkeiten, mit gesundheitsrelevanten Informationen umzugehen, um gesundheitliche Belastungen und Krankheiten zu bewältigen. Chronisch Kranke haben in dieser Untersuchung im Vergleich zu Gesunden noch deutlich häufiger Schwierigkeiten beim Umgang mit Gesundheitsinformationen, d.h. sie sind infolge von deren Komplexität mit der Beurteilung völlig überfordert [1]. Die aktuell publizierte Studie "Euroaspire", in der über 8'200 Herzpatienten aus 81 Regionen von 27 Ländern Europas untersucht und zu persönlichen Risikofaktoren befragt wurden, zeigt ergänzend dazu, dass Patienten bei der Prävention offenbar oft sich selbst überlassen werden. Immerhin 19 Prozent der Patienten gaben 6 Monate nach dem Ereignis an, weiter zu rauchen, 82 Prozent waren übergewichtig, und 34 Prozent bewegten sich zu wenig. Die Mehrheit der Raucher hatte nie versucht, mit dem Rauchen aufzuhören und hatte es auch zukünftig nicht vor. Jeder sechste Raucher gab an, noch nie Hilfe bei der Rauchentwöhnung angeboten bekommen zu haben, fast jeder Zweite hatte nach eigener Aussage noch nie Ratschläge zur Integration von Bewegung in den Alltag erhalten [2].

Diese Studienergebnisse sind leider für in der Patientenversorgung tätige Ärzte alles andere als überraschend. Seit mehreren Jahren richten sich deshalb viele der in den deutschen medizinischen Leitlinien empfohlenen Maßnahmen zur primären bis tertiären Prävention bei chronischen Krankheiten nicht an die Betroffenen, sondern an Politik und Industrie. Dennoch ist gerade Deutschland bei der Prävention im Gegensatz zu vielen anderen Staaten in Europa und sogar weltweit noch immer ein Entwicklungsland. Die Bundesregierung setzt z.B. im Bereich der Ernährung weiterhin hauptsächlich auf Programme für Ernährungsbildung und auf freiwillige Vereinbarungen mit der Industrie. Von den wissenschaftlichen Experten empfohlene verhältnispräventive Maßnahmen werden dagegen größtenteils nicht umgesetzt, die vorhandene Evidenz wird ignoriert, angezweifelt oder sogar lächerlich gemacht [3]. So zielt auch das nach mehreren Jahren des Kampfes endlich ratifizierte Präventionsgesetz nur auf eine Veränderung des individuellen Verhaltens [3], obwohl wissenschaftlich längst belegt ist, dass verhaltenspräventive Ansätze als alleiniger Weg zum Scheitern verurteilt sind, was kürzlich z.B. bei adipösen Schulkindern gezeigt wurde [4].

Gesunde oder Patienten mit dem Willen zur Prävention müssen sich in Deutschland zudem mit den Problemen Qualität und Verfügbarkeit der Angebote, ökono-

\section{KARGER}

(C) 2019 S. Karger AG, Basel 
mische Interessen und Unabhängigkeit von Empfehlungen auseinandersetzen, wobei sie diese Probleme oft nicht einmal wahrnehmen bzw. in ihrer Tragweite beurteilen können. So gibt es zwar seit einigen Jahren eine Zentrale Prüfstelle Prävention (ZPP) der gesetzlichen Krankenkassen, die Präventionskurse zu Bewegung, Ernährung, Stressbewältigung/Entspannung und Suchtmittelkonsum zertifiziert, die Vergütung der Krankenkassen für zertifizierte Präventionsleistungen der in letzter Zeit deutlich abnehmenden Zahl von gut dafür qualifizierten Fachkräften wie z.B. den Physiotherapeuten ist jedoch unzureichend. Eine flächendeckende Versorgung kann deshalb schon jetzt nicht mehr realisiert werden. Dafür werden aber inzwischen über 100'000 nicht zertifizierte kostenpflichtige Gesundheits-Apps angeboten, deren Qualität vom Käufer bzw. Nutzer kaum beurteilt werden kann. Erst Anfang 2018 hat die ZPP Richtlinien herausgegeben, nach denen digitale Maßnahmen im Präventionssektor zertifiziert werden können. Es ist davon auszugehen, dass Präventions-Apps, die diese Kriterien erfüllen, eine Zertifizierung erhalten, was bedeuten kann, dass gesetzliche Krankenversicherungen die Gebühren für diese App auch erstatten. Das scheint zunächst ein Vorteil zu sein, denn ein Physiotherapeut (oder auch ein anderer Gesundheitsanbieter) erreichte mit seinen durch die ZPP zertifizierten Präventionsmaßnahmen in seiner Praxis bisher vielleicht 100 oder 200 Menschen, eine App hat dagegen grundsätzlich das Potential des Angebots einer Präventionsmaßnahme für Tausende bis Hunderttausende von Nutzern. Da der direkte Kontakt zwischen Betreuer und Nutzer jedoch für eine personalisierte Prävention extrem wichtig ist und umsetzbar sein muss, ergibt sich hier eine enorme logistische Herausforderung.

Ob bereits Apps mit typischen naturheilkundlichen oder komplementärmedizinischen Verfahren zertifiziert wurden, entzieht sich ebenso meiner Kenntnis wie der Grad der Unabhängigkeit des Vorgangs der Zertifizierung bei der ZPP. Zudem ist der Inhalt einer Gesundheits-App dem behandelnden Arzt in der Regel nicht genau bekannt, wenn er denn überhaupt von deren Anwendung erfährt. Ärzte befinden sich hier in der gleichen Situation wie bei den frei verkäuflichen Arzneimitteln und insbesondere den stark bei den Verbrauchern beworbenen Nahrungsergänzungsmitteln, deren unkritische und unkontrollierte Anwendung den Patienten bekanntlich durchaus gefährden kann.

Die Verbreitung gesundheitsrelevanter Informationen für die Bevölkerung war früher eine wesentliche Aufgabe von medizinischen Fachgesellschaften und von Vereinen, die sich um ein spezielles Gebiet kümmerten, z.B. auch den Naturheilvereinen. Auch heutzutage verlassen sich Patienten nicht nur darauf, was Ärzte diagnostizieren oder zur Therapie empfehlen, zumal sie deutlich wahrnehmen, dass Ärzte kaum Zeit für ausführliche Gespräche haben. Nach einer aktuellen Studie informieren sich 58 Prozent der befragten Internet-Nutzer vor einem Arztbesuch online, 62 Prozent recherchieren nach dem Arzttermin noch weiter [5]. "Dr. Google" gibt jedoch angepasste Antworten in Form eines sich stets verändernden Algorithmus aus. Diese Informationen zu sondieren und zu sortieren ist für die Nutzer keine leichte Aufgabe, d.h. sie müssen viel Zeit investieren und finden gegebenenfalls dennoch keine unabhängigen Informationen ohne kommerziellen Hintergrund. Das gilt auch für naturheilkundliche und komplementärmedizinische Inhalte.

Infolge der starken Ökonomisierung des deutschen Gesundheitswesens und der derzeitigen Geringschätzung der "sprechenden" Medizin ist es also derzeit selbst bei besten Vorsätzen schon für Gesunde eine erhebliche Herausforderung, die eigene Gesundheitskompetenz zu verbessern und Prävention zu betreiben. Um für Patienten mit chronischen Erkrankungen zukünftig eine wirksame Prävention zu gewährleisten, werden wegen der variierenden Biografien, Bedürfnisse und Präferenzen jedoch insbesondere nutzerzentrierte Informationen benötigt. Dies gilt z.B. auch für die Planung, Entwicklung und Implementierung von digitalen Gesundheitsanwendungen. Am besten kann dieser sehr hohe Anspruch erfüllt werden, wenn die diesbezügliche translationale Forschung gestärkt wird und der reibungslose, schnelle Transfer wissenschaftlicher Erkenntnisse in die Patientenversorgung optimiert wird. Hierzu müssen Lehre und klinische Ausbildung in den betreffenden Fächern einschließlich der Naturheilkunde/integrativen Medizin an universitären und außeruniversitären Einrichtungen jedoch dringend flächendeckend etabliert bzw. ausgebaut werden.

Die aktualisierte S3-Leitlinie zur Colitis ulcerosa enthält gute Beispiele dafür, dass wirksame, den Patientenpräferenzen entgegenkommende naturheilkundliche Therapien, die teilweise auch für das Selbstmanagement geeignet sind bzw. präventiven Charakter haben, zur Verfügung stehen [6]. Da die Deutsche Gesellschaft für Naturheilkunde im November 2018 in die Arbeitsgemeinschaft der Medizinischen Wissenschaftlichen Fachgesellschaften (AWMF) aufgenommen wurde, wird die Zahl der Leitlinien, die Aussagen zur Evidenzbasierung von naturheilkundlichen und komplementärmedizinischen Verfahren enthalten, zukünftig stark ansteigen. Dies ist auch ein wichtiger Schritt für eine Stärkung der Gesundheitskompetenz gerade chronisch Kranker in Deutschland, da sie sich in den Leitlinien rasch, wenn auch nicht immer ganz unabhängig von ökonomischen Interessen, informieren können. Kontraproduktives Verhalten von Patienten, wie es in der zu Anfang zitierten Studie dokumentiert wurde [2], ist nämlich nur dann zu akzeptieren, wenn sie sich trotz ausreichender Informationen freiwil- 
lig dafür entschieden haben. Freier Patientenwille und mangelnde oder nicht unabhängige Informationsmöglichkeiten sind dagegen nicht miteinander vereinbar. Die deutsche Gesundheitspolitik ist deshalb dringend gefordert, die Experten, die unabhängige Informationen erarbeiten können, endlich staatlich zu unterstützen und das Gesundheitssystem entsprechend zu reformieren. Viele andere Länder gehen hier seit Jahren mit gutem Beispiel und sehr erfolgreich voran.

\section{Disclosure Statement}

Die Autorin gibt keine Interessenskonflikte an.

\section{Literatur}

1 Schaeffer D, Vogt D, Berens EM, Hurrelmann K. Gesundheitskompetenz der Bevölkerung in Deutschland. Ergebnisbericht 2016. www. uni-bielefeld.de/gesundhw/ag6/downloads/ Ergebnisbericht_HLS-GER.pdf; Stand: 1.3.2019.

2 Kotseva K, De Backer G, De Bacquer D, Rydén L, Hoes A, Grobbee D, Maggioni A, Marques-Vidal P, Jennings C, Abreu A, Aguiar C, Badariene J, Bruthans J, Castro Conde A, Cifkova R, Crowley J, Davletov K, Deckers J, De Smedt D, De Sutter J, Dilic M, Dolzhenko M, Dzerve M, Erglis A, Fras Z, Gaita D, Gotcheva N, Heuschmann P, Hasan-Ali H, Jankowski P, Lalic N, Lehto S, Lovic D, Mancas S, Mellbin L, Milicic D, Mirrakhimov E,
Oganov R, Pogosova N, Reiner Z, Stöerk S, Tokgözoğlu L, Tsioufis C, Vulic D, Wood D, on behalf of the EUROASPIRE Investigators. Lifestyle and impact on cardiovascular risk factor control in coronary patients across 27 countries: Results from the European Society of Cardiology ESC-EORP EUROASPIRE V registry. Eur J Prev Cardiol. 2019 Feb 10: 2047487318825350.

3 https://www.deutsche-diabetes-gesellschaft. $\mathrm{de} /$ presse/ddg-pressemeldungen/meldungen-detailansicht/article/praeventionsgesetzgeht-an-den-wirklichen-problemen-vorbeideutsche-diabetes-gesellschaft-fordert-a.html

4 Adab P, Pallan MJ, Lancashire ER, Hemming K, Frew E, Barrett T, et al. Effective- ness of a childhood obesity prevention programme delivered through schools, targeting 6 and 7 year olds: cluster randomised controlled trial (WAVES study). BMJ. 2018 Feb;360:k211.

5 https://www.bertelsmann-stiftung.de/de/ publikationen/publikation/did/nutzungund-verbreitung-von-gesundheitsinformationen/

6 Kucharzik T, Dignass AU, Atreya R, Bokemeyer B, Esters P, Herrlinger K, et al. Aktualisierte S3-Leitlinie Colitis ulcerosa der Deutschen Gesellschaft für Gastroenterologie, Verdauungs- und Stoffwechselkrankheiten (DGVS) - AWMF-Register-Nr. 021/009. Z Gastroenterol. 2018 Sep;56(9):1087-169. 